\title{
Synchronization in output-coupled temporal Boolean networks
}

SUBJECT AREAS: COMPLEX NETWORKS APPLIED MATHEMATICS

Received

23 May 2014

Accepted

19 August 2014

Published

5 September 2014

Correspondence and requests for materials should be addressed to J.L. (jqluma@seu.edu.
Jianquan Lu', Jie Zhong' ${ }^{1}$ Y Yang Tang ${ }^{2,3}$, Tingwen Huang ${ }^{4}$, Jinde Cao ${ }^{1,5}$ \& Jürgen Kurths ${ }^{2,3,6}$

${ }^{1}$ Department of Mathematics, Southeast University, Nanjing 210096 , China, ${ }^{2}$ Potsdam Institute for Climate Impact Research, Potsdam 14415, Germany, ${ }^{3}$ Institute of Physics, Humboldt University of Berlin, Berlin 12489, Germany, ${ }^{4}$ Texas A\&M University at Qatar, Doha 23874, Qatar, ${ }^{5}$ Department of Mathematics, Faculty of Science, King Abdulaziz University, Jeddah 21589, Saudi Arabia,

'Department of Control Theory, Nizhny Novgorod State University, Gagarin Avenue 23, 606950 Nizhny Novgorod, Russia.

This paper presents an analytical study of synchronization in an array of output-coupled temporal Boolean networks. A temporal Boolean network (TBN) is a logical dynamic system developed to model Boolean networks with regulatory delays. Both state delay and output delay are considered, and these two delays are assumed to be different. By referring to the algebraic representations of logical dynamics and using the semi-tensor product of matrices, the output-coupled TBNs are firstly converted into a discrete-time algebraic evolution system, and then the relationship between the states of coupled TBNs and the initial state sequence is obtained. Then, some necessary and sufficient conditions are derived for the synchronization of an array of TBNs with an arbitrary given initial state sequence. Two numerical examples including one epigenetic model are finally given to illustrate the obtained results.

central focus of modern biology concerns understanding the function of a cell, the manner how a cell performs its function and the ways in which cellular systems fail in disease. With the rapid development of systems biology ${ }^{1,2}$, genetic regulatory networks have recently become a newly developing area and have attracted much attention due to its close relationship with neural networks, protein webs and other biological systems $^{3-7}$. A genetic regulatory network contains a group of DNA elements in a cell which interact with others directly or indirectly through their mRNA or protein products. Thus, a genetic regulatory network governs the expression levels of the mRNA and the protein products. With time going, various types of genetic regulatory networks have been proposed, such as Markov-type genetic networks ${ }^{8}$ and Boolean networks ${ }^{1}$. Each of these models has its own advantages and limitations in terms of the following considerations: the accuracy of the approach relate to the level of genetic, biological phenomenon being modelled; the experimental feasibility of model construction; and the computational complexity of the model inference from the available data.

The concept of Boolean network (BN) was firstly proposed by Kauffman ${ }^{1}$ as a simplified model of the behavior of large networks of randomly interconnected binary (on-off) genes. One of the main advantages of Boolean networks is the comprehensibility owing to the simplicity of the representation. A BN can effectively model a genetic regulatory network with its gene products (the outputs) and the substances from outside or the environment that can affect the gene (the inputs). In a BN, the state of each node has only two unique states: 1 (on) or 0 (off). For a gene, "on" corresponds to the gene being expressed; For inputs and outputs, "on" corresponds to the relative substances being present. Moreover, each node evolves its state according to a Boolean function, which is a logical function, at each discrete time point.

Since the BNs can provide a general description of the behavior of many living organisms, they have attracted great attention from researchers in different fields. There are various examples of genetic regulatory networks modeled using BNs, such as the yeast cell-cycle networks; the control of the mammalian cell cycle ${ }^{10}$; the yeast transcriptional networks ${ }^{11}$; the network predicting the expression of the segment polarity genes in the fly Drosophila melanogaster ${ }^{12}$, and so on. Recently, a new method called semi-tensor product (STP) of matrices has been firstly proposed by Cheng and his colleagues ${ }^{13,14}$. By using the method of STP, a Boolean function can be uniquely expressed as an algebraic expression, and hence a BN can be expressed as a standard discrete-time algebraic system. This method has been proved to be quite useful to study BNs concerning the topological structure, such as the fixed points, cycles, basin of attractors and the transient time. Based on this approach, many interesting results have been obtained in the last few years, such as stabilization of BNs with impulsive effects $^{15}$, optimal control of logical control networks ${ }^{16}$, etc.

A curious ability of some real-world systems is that they can evolve in perfect synchronization. In the past decades, the phenomenon of collective behavior has inspired a large amount of research, such as synchronization analysis and control of complex networks ${ }^{17-21}$, consensus in multi-agent systems ${ }^{22,23}$, synchronization of 
Kauffman networks ${ }^{24}$, and cooperation of networks ${ }^{25-27}$, etc. It was demonstrated that the nodes in a second-order Kuramoto model perform a cascade of transitions toward a synchronous macroscopic state $^{28}$. A time delay, which is a source of instability, exists in various engineering systems such as chemical processes, or long transmission lines in pneumatic systems. Some interesting results have also been obtained concerning the effect of time-delay on network synchronization $^{29-31}$. A processing coupling-delay is effectively used to annihilate the quenching of oscillation in a network of coupled oscillators by switching the stability of amplitude death ${ }^{32}$. Recently, interests have also been extended to synchronization of BNs, due to their potential applications in biology, chemistry, as well as engineering $^{33,34}$.

Synchronization and controllability are structural properties of a system, and they are fundamental concepts in control theory. Recently, synchronization of two delay-free deterministic BNs and synchronization in an array of coupled BNs have been respectively studied in $^{33,34}$ and the synchronization of multi-valued logical networks have been studied $\mathrm{in}^{35}$. $\mathrm{In}^{36}$, we investigated the synchronization in an array of BNs with time delay. It is well known that time delay is very common in real world and also is inevitable in biological systems and engineering problems. Moreover, in many situations, time delay can not be ignored in practice, since it may heavily affect the dynamics of the networks ${ }^{37,38}$. One interesting kind of BNs, called temporal Boolean networks (TBNs) were developed to model regulatory delays $\mathrm{in}^{39}$. TBNs own a more complex structure when the time delays in Boolean variables are allowed to be different. TBNs can approximate real cellular regulatory networks or genetic networks better than BNs. Hence, it is desirable to study synchronization of TBNs. In the past few years, many results about TBNs have been obtained, such as output controllability of temporal Boolean control networks (TBCNs) and complete synchronization of $\mathrm{TBNs}^{40}$. However, there has been no results investigating synchronization in an array of output-coupled TBNs, to the best of our knowledge. $\mathrm{In}^{40}$, the authors studied the synchronization of two coupled TBNs in a drive-response configuration. Sometimes, in the real world, we need to judge whether an array of networks can be synchronized or not. The output coupling phenomenon is ubiquitous in many biological networks and physical systems. For instance, a cellular system contains many different components to synthesis macromolecular in cells. In an idealized model of a metabolic feed-back control cycle, each unit component consists of three parts: a genetic locus, a ribosome and a cellular locus. Each of these three parts interacts with each other by its corresponding products, such as mRNA, protein and metabolic species. Furthermore, different components interacts with each other by its corresponding metabolic products, which is a type of output coupling. Thus, investigating synchronization in an array of output-coupled TBNs is meaningful and challenging, and can be helpful to disclose the control mechanisms for macromolecular synthesis and the behavior of a cell.

Motivated by the above discussions, in this paper, we focus on synchronization in an array of output-coupled TBNs. Our main objective is to obtain a general analytical approach to study synchronization in output-coupled TBNs. Both state delay and output delay are considered in the output-coupled TBNs. The main results are derived by using the method of semi-tensor product of matrices which can convert a deterministic $\mathrm{BN}$ into a unique algebraic representation. By using the algebraic representations of TBNs, we derive some necessary and sufficient conditions for the synchronization in an array of TBNs.

Some basic notations: The standard notations will be used in this paper. Throughout this paper, the notation $\mathbf{1}_{k}$ denotes the $k$ dimensional column vector with all entries equalling to 1 . Denote the set of two logical variables by $\mathcal{D}=\{1,0\}$, where $1 \sim T$ means "true" and $0 \sim F$ means "false". A logical variable $u$ will take a value from $\mathcal{D}$, which is expressed as $u \in \mathcal{D}$. Define a delta set as
$\Delta_{n}:=\left\{\delta_{n}^{i} \mid i=1,2, \ldots, n\right\}$, where $\delta_{n}^{i}$ is the $i$ th column of the identity matrix $I_{n}$. We denote the $i$ th column of matrix $A$ by $\operatorname{Col}_{i}(A)$ and denote the set of columns of matrix $A$ by $\operatorname{Col}(A)$. We also denote the $i$ th row of matrix $A$ by $\operatorname{Row}_{i}(A)$ and denote the set of rows of matrix $A$ by $\operatorname{Row}(A)$. An $n \times m$ matrix $L$ is called a logical matrix if the columns of $L$, denoted by $\operatorname{Col}(L)$, are in the form of $\delta_{n}^{k}$. That is to say, $\operatorname{Col}(L) \subseteq \Delta_{n}$. Denote the set of $n \times s$ logical matrices by $\mathcal{L}_{n \times s}$. If $L \in L_{n \times s}$, it can be expressed as $L=\left[\delta_{n}^{i_{1}}, \delta_{n}^{i_{2}}, \ldots, \delta_{n}^{i_{s}}\right]$. For simplicity, it can be denoted as $L=\delta_{n}\left[i_{1}, \ldots, i_{s}\right]$. We use $\otimes_{i=1}^{M} X_{i}(t)$ to denote $X_{1}(t) \otimes X_{2}(t) \otimes \cdots \otimes X_{M}(t), \quad$ and use $\times_{j=1}^{N} y_{i}(t)$ to denote $y_{1}(t) \ltimes y_{2}(t) \ltimes \cdots \ltimes y_{N}(t)$.

\section{Results and Methods}

In this paper, we study the following array of $M$ output-coupled BNs, with each one being an $N$-node system:

$$
\left\{\begin{aligned}
X_{j}^{i}(t+1)= & f_{j}^{i}\left(X_{j}^{1}(t), \ldots, X_{j}^{N}(t), X_{j}^{1}(t-1), \ldots, X_{j}^{N}(t-1), \ldots,\right. \\
& \left.X_{j}^{1}\left(t-\tau_{1}\right), \ldots, X_{j}^{N}\left(t-\tau_{1}\right), y_{1}(t), \ldots, y_{M}(t)\right), \\
& i=1,2, \ldots, N \\
y_{j}(t)= & g_{j}\left(X_{j}^{1}(t), \ldots, X_{j}^{N}(t), X_{j}^{1}(t-1), \ldots, X_{j}^{N}(t-1), \ldots,\right. \\
& X_{j}^{1}\left(t-\tau_{2}+1\right), \ldots, X_{j}^{N}\left(t-\tau_{2}+1\right), X_{j}^{1}\left(t-\tau_{2}\right), \ldots, \\
& \left.X_{j}^{N}\left(t-\tau_{2}\right)\right), j=1,2, \ldots, M
\end{aligned}\right.
$$

where $X_{j}^{i}$ is the $i$ th node of the $j$ th TBN, $y_{j}$ is the binary output, $f_{j}^{i}:\{1,0\}^{\left(\tau_{1}+1\right) N+M} \rightarrow\{1,0\}, g_{j}:\{1,0\}^{\left(\tau_{2}+1\right) N} \rightarrow\{1,0\}$ are Boolean functions, and $\tau_{1}, \tau_{2}$ are positive integers describing the state delay and the output delay. In many biological networks and genetic regulatory networks, time delay is ubiquitous due to finite signal propagation time, finite chemical reaction times, memory effects, and so on. The time delay $\tau_{1}$ describes delay occurring between states, while time delay $\tau_{2}$ describes delay resulting from output. For example, in a genetic regulatory networks, the level of a gene maybe affected by its past $\mu$ gene levels. Different genes interact by its corresponding gene products, which may cost time to be transmitted from one gene to another one. Thus, model (1) can be an efficient model to describe some biological networks or genetic regulatory networks with state delay and output delay. We simply denote $X_{j}(t)=\left(X_{j}^{1}(t), X_{j}^{2}(t), \ldots, X_{j}^{N}(t)\right)^{T}$ the states of the TBNs (1) at time instant $t$. We observe that the state evolution of the output-coupled TBN (1) depends on the initial state sequences, and there are two cases as follows. Case (I): $\tau_{1} \geq \tau_{2}$, the initial state sequence is $X_{j}(0)$, $X_{j}(-1), \ldots, X_{j}\left(-\tau_{1}\right)$ for $j=1,2, \ldots, M$; case (II): $\tau_{1}<\tau_{2}$, the initial state sequence is $X_{j}(0), X_{j}(-1), \ldots, X_{j}\left(-\tau_{2}\right)$ for $j=1,2, \ldots, M$. Now, we define complete synchronization in the array of TBNs (1) as follows:

Definition 1 The array of TBNs are said to be synchronized if for every initial state sequence, there exists a positive integer $k$, such that $t$ $\geq k$ implies $X_{i}(t)=X_{j}(t)$ for every distinct $1 \leq i, j \leq M$.

Remark 1 In this definition, the value of $k$ depends on the initial state sequences. Nevertheless, since the $\{1,0\}^{\left(\tau_{1}+1\right) N}\left(\right.$ or $\left.\{1,0\}^{\left(\tau_{2}+1\right) N}\right)$ is a finite set, we can always choose a constant $k$ large enough which is independent of the initial states sequence.

In the following, we establish some necessary and sufficient criteria for synchronization in an array of output-coupled TBNs. To acquire a strict analysis, we ought to apply algebraic representations of the TBNs according to the STP theory.

Let $X_{j}(t)=X_{j}^{1}(t) \ltimes X_{j}^{2}(t) \ltimes \cdots \ltimes X_{j}^{N}(t)$ for $j=1, \ldots, M$ and $y(t)=y_{1}(t) \ltimes y_{2}(t) \ltimes \cdots \ltimes y_{M}(t)$. Let $F_{j}^{i}$ and $G_{j}$ be the structure matrix 
of $f_{j}^{i}$ and $g_{j}$. Then we can make $A_{j}\left(t, \tau_{2}\right)=$ $X_{j}(t) X_{j}(t-1) \cdots X_{j}\left(t-\tau_{2}\right)$ for $j=1,2, \ldots, M$ to obtain the algebraic representations of $y(t)$. By equation (1) and the algebraic representations of Boolean functions, we have

$$
y_{j}(t)=G_{j} X_{j}(t) X_{j}(t-1) \cdots X_{j}\left(t-\tau_{2}\right)=G_{j} A_{j}\left(t, \tau_{2}\right) .
$$

Hence, we get $y(t)=\left(\otimes_{j=1}^{M} G_{j}\right) \ltimes_{j=1}^{M} A_{j}\left(t, \tau_{2}\right) \triangleq G \ltimes_{j=1}^{M} A_{j}\left(t, \tau_{2}\right)$, where $G=\otimes_{j=1}^{M} G_{j}$. To obtain the accurate algebraic expressions of $\ltimes_{j=1}^{M} A_{j}\left(t, \tau_{2}\right)$, we also make $M_{j}\left(t, \tau_{2}\right)=X_{j}(t-1) \cdots X_{j}\left(t-\tau_{2}\right)$ to facilitate the expression. Then we have

$$
\begin{aligned}
\ltimes_{j=1}^{M} A_{j}(t)= & X_{1}(t) X_{1}(t-1) \cdots X_{1}\left(t-\tau_{2}\right) X_{2}(t) \cdots X_{2}\left(t-\tau_{2}\right) \\
& \cdots X_{M}(t) \cdots X_{M}\left(t-\tau_{2}\right) \\
= & X_{1}(t) M_{1}\left(t, \tau_{2}\right) X_{2}(t) M_{2}\left(t, \tau_{2}\right) X_{3}(t) M_{3}\left(t, \tau_{2}\right) \\
& \cdots X_{M}(t) M_{M}\left(t, \tau_{2}\right) \\
= & X_{1}(t) W_{\left[2^{N}, 2^{\tau} 2^{N}\right]} X_{2}(t) M_{1}\left(t, \tau_{2}\right) M_{2}\left(t, \tau_{2}\right) X_{3}(t) \\
& M_{3}\left(t, \tau_{2}\right) \cdots X_{M}(t) M_{M}\left(t, \tau_{2}\right) \\
= & \left(I_{2^{N}} \otimes W_{\left[2^{N}, 2^{\tau} 2^{N}\right]}\right) X_{1}(t) X_{2}(t) M_{1}\left(t, \tau_{2}\right) M_{2}\left(t, \tau_{2}\right) \\
& X_{3}(t) M_{3}\left(t, \tau_{2}\right) \cdots X_{M}(t) M_{M}\left(t, \tau_{2}\right) \\
& \vdots \\
= & \ltimes_{i=1}^{M-1}\left(I_{2^{i N}} \otimes W_{\left[2^{N}, 2^{i \tau_{2} N}\right]}\right)\left(\ltimes_{j=1}^{M} X_{j}(t)\right) M_{1}\left(t, \tau_{2}\right) \\
& M_{2}\left(t, \tau_{2}\right) \cdots M_{M}\left(t, \tau_{2}\right) \\
\triangleq & W_{0}\left(\ltimes_{j=1}^{M} X_{j}(t)\right) M_{1}\left(t, \tau_{2}\right) M_{2}\left(t, \tau_{2}\right) \cdots M_{M}\left(t, \tau_{2}\right) \\
= & W_{0}\left(\ltimes_{j=1}^{M} X_{j}(t)\right) X_{1}(t-1) \cdots X_{1}\left(t-\tau_{2}\right) \cdots X_{M}(t-1) \\
& \cdots X_{M}\left(t-\tau_{2}\right),
\end{aligned}
$$

where $W_{0}=\ltimes_{i=1}^{M-1}\left(I_{2^{i N}} \otimes W_{\left[2^{N}, 2^{i 2_{2} N}\right]}\right)$. By induction, we yield

$\ltimes_{j=1}^{M} A_{j}\left(t, \tau_{2}\right) \triangleq \widehat{W}_{1} \ltimes_{j=1}^{M} X_{j}(t) \ltimes_{j=1}^{M} X_{j}(t-1) \cdots \ltimes_{j=1}^{M} X_{j}\left(t-\tau_{2}\right)$,

where $\widehat{W}_{1}=W_{0} \ltimes_{j=1}^{\tau_{2}-1}\left(I_{2^{j N} M} \otimes W_{j}\right), W_{j}=\ltimes_{i=1}^{M-1}\left(I_{2^{i N}} \otimes W_{\left[2^{N}, 2^{i N\left(\tau_{2}-j\right)}\right]}\right), j$ $=1, \ldots, \tau_{2}-1$. Hence, we have $y(t)=G \widehat{W}_{1} \ltimes_{j=1}^{M} X_{j}(t) \ltimes_{j=1}^{M}$ $X_{j}(t-1) \cdots \ltimes_{j=1}^{M} X_{j}\left(t-\tau_{2}\right)$.

Now, we try to obtain the algebraic representations of the states $X_{j}(t+1)$. By equation (1) and algebraic representations of Boolean functions, we have $X_{j}^{i}(t+1)=F_{j}^{i} X_{j}(t) X_{j}(t-1) \cdots X_{j}\left(t-\tau_{1}\right) y(t)$. By denoting $C\left(t, \tau_{1}\right)=X_{j}(t) X_{j}(t-1) \cdots X_{j}\left(t-\tau_{1}\right) y(t)$ for simplicity, we then get

$$
\begin{aligned}
X_{j}(t+1) & =F_{j}^{1} C\left(t, \tau_{1}\right) F_{j}^{2} C\left(t, \tau_{1}\right) \cdots F_{j}^{N} C\left(t, \tau_{1}\right) \\
& =F_{j}^{1}\left(I_{2^{\left(\tau_{1}+1\right)}+M} \otimes F_{j}^{2}\right) \Phi_{\left(\tau_{1}+1\right) N+M} C\left(t, \tau_{1}\right) \cdots F_{j}^{N} C\left(t, \tau_{1}\right)(5) \\
& \triangleq \\
& F_{j} C\left(t, \tau_{1}\right)=F_{j} X_{j}(t) X_{j}(t-1) \cdots X_{j}\left(t-\tau_{1}\right) y(t) .
\end{aligned}
$$

where $F_{j}=F_{j}^{1} \ltimes_{j=2}^{N}\left\{\left(I_{2^{\left(\tau_{1}+1\right) N+M}} \otimes F_{j}^{i}\right) \Phi_{\left(\tau_{1}+1\right) N+M}\right\}$.

That is to say, we have obtained the following equivalent algebraic representations of output-coupled TBNs (1):

$$
\left\{\begin{array}{l}
X_{j}(t+1)=F_{j} X_{j}(t) X_{j}(t-1) \cdots X_{j}\left(t-\tau_{1}\right) y(t) \\
y(t)=G \widehat{W}_{1} \ltimes_{j=1}^{M} X_{j}(t) \ltimes_{j=1}^{M} X_{j}(t-1) \cdots \ltimes_{j=1}^{M} X_{j}\left(t-\tau_{2}\right)
\end{array}\right.
$$

where $F_{j}$ is an $2^{N} \times 2^{\left(\tau_{1}+1\right) N+M}$ matrix, $G$ is an $2^{M} \times 2^{\left(\tau_{2}+1\right) N M}$ matrix and $\widehat{W}_{1}$ is an $2^{\left(\tau_{2}+1\right) N M} \times 2^{\left(\tau_{2}+1\right) N M}$ matrix.

From equation (6), it is easy to see that the state of each TBN is determined by its delayed states and the output $y(t)$, which means these TBNs are coupled through the output $y(t)$. Let $\ltimes_{j=1}^{M} X_{j}(t)=X_{1}(t) \ltimes X_{2}(t) \ltimes \cdots \ltimes X_{M}(t)$, using equation (6) and mathematical iteration, we can derive the relationship between $\ltimes_{j=1}^{M} X_{j}(t)$ and corresponding initial state sequence.

Before we derive the necessary and sufficient conditions for synchronization, we need the following proposition. From this proposition, we can acquire the relationship between the state $X_{j}(t)$ at time $t$ and the initial state sequence which will be employed to obtain the criteria for synchronization of TBNs.

Proposition 1 Let $F=\otimes_{j=1}^{M} F_{j}, \quad W=W_{\left[2^{M}, 2^{\left(\tau_{1}+1\right) N}\right]} \aleph_{i=2}^{M}$ $\left\{\left(I_{2^{M}} \otimes W_{\left[2^{M}, 2^{i\left(\tau_{1}+1\right) N}\right]}\right) \Phi_{M}\right\} \quad$ and $\quad \widehat{W}_{2}=W^{\prime}{ }_{0} \ltimes_{j=1}^{\tau_{1}-1}\left(I_{2^{j M N}} \otimes W^{\prime}{ }_{j}\right)$, where $W^{\prime}{ }_{j}=\ltimes_{i=1}^{M-1}\left(I_{2^{i N}} \otimes W_{\left[2^{N}, 2^{i\left(\tau_{1}-j\right) N}\right]}\right), j=0,1, \ldots, \tau_{1}-1$.

Then we have

$$
\begin{aligned}
& \ltimes_{j=1}^{M} X_{j}(t+1)=F W G \widehat{W}_{1}\left(I_{2^{\left(\tau_{2}+1\right) N M}} \otimes \widehat{W}_{2}\right) \ltimes_{j=1}^{M} X_{j}(t) \ltimes_{j=1}^{M} X_{j}(t-1) \\
& \cdots \ltimes_{j=1}^{M} X_{j}\left(t-\tau_{2}\right) \ltimes_{j=1}^{M} X_{j}(t) \ltimes_{j=1}^{M} X_{j}(t-1) \\
& \cdots \ltimes_{j=1}^{M} X_{j}\left(t-\tau_{1}\right) \text {. }
\end{aligned}
$$

Remark 2 According to equation (7), we need to know whether $\tau_{1}$ is larger than $\tau_{2}$ or not by considering these two cases: Case (I): $\tau_{1} \geq \tau_{2}$, which means that the initial state sequence is $X_{j}(0), \ldots, X_{j}\left(-\tau_{1}\right)$ for $j=$ 1, 2, .., M; Case (II): $\tau_{1}<\tau_{2}$, which implies the initial state sequence is $X_{j}(0), \ldots, X_{j}\left(-\tau_{2}\right)$ for $j=1,2, \ldots, M$. In the following sequel, according to different case, we derive some necessary and sufficient conditions for synchronization in an array of output-coupled TBNs. We firstly consider Case (I), and then Case (II).

Case I: $\tau_{1} \geq \tau_{2}$. Now let us first consider Case (I): $\tau_{1} \geq \tau_{2}$, with the initial state $X_{j}(0), \ldots, X_{j}\left(-\tau_{1}\right)$ for $j=1,2, \ldots, M$. Since $\tau_{1}$ and $\tau_{2}$ describe the state delay and the output delay, in this case, it means the state delay is not smaller than the output delay. In a cellular system, it means the delay caused during the processes of the transcription of a gene is not smaller than that caused during the processes of the translation of a gene. Based on Proposition 1, we obtain the following proposition, which is derived to show the explicit relationship between the state $X_{j}(t)$ and the initial states $X_{j}(0), \ldots$, $X_{j}\left(-\tau_{1}\right)$.

Proposition 2 Let $\Theta_{1}=F W G \widehat{W}_{1}\left(I_{2^{\left(\tau_{2}+1\right) N M}} \otimes \widehat{W}_{2}\right) \Phi_{\left(\tau_{2}+1\right) N M}$ and $\Xi_{1}=\Theta_{1} W_{\left[2^{\tau_{1} N M}, 2^{\left(\tau_{1}+1\right) N M}\right]} \Phi_{\tau_{1} N M}$, then we have

$$
\ltimes_{j=1}^{M} X_{j}(t+1)=\Theta_{1} \ltimes_{j=1}^{M} X_{j}(t) \ltimes_{j=1}^{M} X_{j}(t-1) \cdots \ltimes_{j=1}^{M} X_{j}\left(t-\tau_{1}\right)
$$

and

$$
\aleph_{j=1}^{M} X_{j}(t)=\left(I_{2^{N M}} \otimes 1_{2^{\tau_{1} N M}}^{T}\right) \Xi_{1}^{t}\left\{\ltimes_{j=1}^{M} X_{j}(0) \ltimes_{j=1}^{M} X_{j}(-1) \cdots \ltimes_{j=1}^{M} X_{j}\left(-\tau_{1}\right)\right\} .
$$

In the first case, i.e. $\tau_{1} \geq \tau_{2}$, which means the state delay is larger than the output delay, we can observe the value of states at time $t+1$ depends on its past $\tau_{1}+1$ states. Then, using mathematical analysis and STP, we can obtain that the value of states at time $t$ has a specific connection with the initial sequence, $\ltimes_{j=1}^{M} X_{j}(0), \ltimes_{j=1}^{M} X_{j}(-1), \ldots$, $\aleph_{j=1}^{M} X_{j}\left(-\tau_{1}\right)$. The left side of equation (9) is the STP of state of each TBN at the discrete time point $t$, while the right side is product of several matrices and the initial state sequences. Since $\left(I_{2^{M N}} \otimes 1_{2^{\tau_{1} N M}}^{T}\right)$ and $\Xi_{1}$ are constant matrices, we can present our necessary and 
sufficient criterion for synchronization in the array of outputcoupled TBNs as following.

Theorem 1 Let equation (6) be the algebraic representations of the array of TBNs (1). Then synchronization occurs iff there exists a positive integer $k$ satisfying $1 \leq k \leq k_{0}$ such that

$\operatorname{Col}\left[\left(I_{2^{N M}} \otimes 1_{2^{\tau_{1} N M}}^{T}\right) \Xi_{1}^{k}\right] \subseteq\left\{\delta_{2^{\lambda_{i}}}^{\lambda_{i}}: \lambda_{i}=1+\frac{(i-1)\left(2^{M N}-1\right)}{2^{N}-1}, i=1,2, \ldots, 2^{N}\right\}$

where

$k_{0}=\min \left\{i \geq 1:\left(I_{2^{N M}} \otimes 1_{2^{\tau_{1} N M}}^{T}\right) \Xi_{1}^{i}=\left(I_{2^{N M}} \otimes 1_{2^{\tau_{1} N M}}^{T}\right) \Xi_{1}^{j}, j>i\right\}$.

Remark 3 In equation (11) in Theorem 1, we can use $k_{0}^{\prime}=\min \left\{i \geq 1: \Xi_{1}^{i}=\Xi_{1}^{j}, j>i\right\}$ to simplify it but meanwhile the restriction is strengthened. By $k_{0}^{\prime}=\min \left\{i>1: \Xi_{1}^{i}=\Xi_{1}^{j}, j>i\right\}$, there must exist an integer $j>k_{0}^{\prime}$ such that $\Xi_{1}^{k_{0}^{\prime}}=\Xi_{1}^{j}$. Then we have $\left(I_{2^{N M}} \otimes 1_{2^{\tau_{1} N M}}^{T}\right) \Xi_{1}^{k_{0}^{\prime}}=\left(I_{2^{N M}} \otimes 1_{2^{\tau_{1} N M}}^{T}\right) \Xi_{1}^{j}$. Hence, we can conclude that $k_{0} \leq k_{0}^{\prime}$. Equation (10) implies that after finite time steps, i.e. $k_{0}$ steps, we can judge whether the array of TBNs can be synchronized or not. If this array of TBNs can not be synchronized before the $k_{0}$-th steps, then it can not be synchronized at any time. Thus, Theorem 1 provides an efficient criterion for synchronization in an array of output-coupled TBNs.

Remark 4 If the coupled TBNs can be synchronized after certain times $k$, then we have $X_{j}^{i}(t)=X_{j^{\prime}}^{i^{\prime}}(t)$ for $1 \leq j \neq j^{\prime} \leq M, 1 \leq i \neq i^{\prime} \leq N$ and $t \geq k$. Since the set of state values is a finite set, i.e. $\left\{\delta_{2^{N}}^{1}, \delta_{2^{N}}^{2}, \ldots, \delta_{2^{N}}^{2^{N}}\right\}$, we can suppose each synchronous state to be $X_{j}^{i}=\delta_{2^{N}}^{i}, 1 \leq j \leq M, 1 \leq i \leq N$. Because of the definition of $\ltimes_{j=1}^{M} X_{j}(t)=X_{1}(t) \ltimes \cdots \ltimes X_{M}(t)$, we can obtain the synchronous set of $\ltimes_{j=1}^{M} X_{j}(t)$, which is $\left\{\delta_{2^{M N}}^{\lambda_{i}}: \lambda_{i}=1+\frac{(i-1)\left(2^{M N}-1\right)}{2^{N}-1}, i=1,2, \ldots, 2^{N}\right\}$. According to equation (9), if we have an initial sequence, i.e. $\ltimes_{j=1}^{M} X_{j}(0) \ltimes_{j=1}^{M} X_{j}(-1) \cdots \ltimes_{j=1}^{M} X_{j}\left(-\tau_{1}\right)=\delta_{2^{N M\left(\tau_{1}+1\right)}}^{i}$, we can obtain $\times_{j=1}^{M} X_{j}(t)=\operatorname{Col}_{i}\left[\left(I_{2^{N M}} \otimes 1_{2^{\tau_{1} N M}}^{T}\right) \Xi_{1}^{t}\right]$. Thus, $\operatorname{Col}\left[\left(I_{2^{N M}} \otimes 1_{2^{\tau_{1} N M}}^{T}\right) \Xi_{1}^{t}\right]$ is the total possible state value of $\propto_{j=1}^{M} X_{j}(t)$. In order to judge whether the coupled TBNs can be synchronized or not, we just need to calculate whether there exists a positive integer $k$ such that all possible state value of $\times_{j=1}^{M} X_{j}(k)$ are in the synchronous set $\left\{\delta_{2^{M N}}^{\lambda_{i}}: \lambda_{i}=1+\frac{(i-1)\left(2^{M N}-1\right)}{2^{N}-1}, i=1,2, \ldots, 2^{N}\right\}$.

As we know, $\Xi_{1}$ is a logical matrix and so is the matrix of $I_{2^{M N}} \otimes 1_{2^{\tau_{1} N M}}^{T}$. Moreover, if $\left(\Xi_{1}\right)_{i j}=1$, then we have $\left(I_{2^{N M}} \otimes 1_{2^{\tau_{1} N M}}^{T}\right) \operatorname{Col}_{j}\left(\Xi_{1}\right)=\operatorname{Col}_{i}\left(I_{2^{N M}} \otimes 1_{2^{\tau_{1} N M}}^{T}\right)$ which means taking the $i$ th column of matrix $I_{2^{N M}} \otimes 1_{2^{\tau_{1} N M}}^{T}$. Then, we can easily derive the following simplified criterion for synchronization in an array of output-coupled TBNs.

Corollary 1 Let $\Omega_{1}=\left\{\left(\lambda_{i}-1\right)^{2^{\tau_{1} N M}}+j: \lambda_{i}=1+\frac{(i-1)\left(2^{N M}-1\right)}{2^{N}-1}\right.$, $\left.i=1,2, \ldots, 2^{N}, j=1, \ldots, 2^{\tau_{1} N M}\right\}$. Then the array of output-coupled TBNs (1) can be synchronized iff there exists a positive integer $k$ such that $\operatorname{Row}_{i}\left(\Xi_{1}^{k}\right)=\underbrace{(0,0, \ldots, 0)}_{2^{\left(\tau_{1}+1\right) N M}}, i \in\left\{1,2, \ldots, 2^{\left(\tau_{1}+1\right) N M}\right\} / \Omega_{1}$.

In Proposition 2, we use the variable substitution $Z_{1}(t)=\ltimes_{j=1}^{M} X_{j}(t) \cdots \ltimes_{j=1}^{M} X_{j}\left(t-\tau_{1}\right)$ to analyze the problem of synchronization in an array of output-coupled TBNs. Based on the variable substitution, we derive the necessary and sufficient criterion for synchronization, i.e. Theorem 1. In the following sequel, we use the mathematical induction without using the variable substitution
$Z_{1}(t)=\ltimes_{j=1}^{M} X_{j}(t) \cdots \ltimes_{j=1}^{M} X_{j}\left(t-\tau_{1}\right)$ to analyze the synchronization problem. According to equation (8), we obtain another form of relationship between the state $X_{j}(t)$ and the initial state sequence $X_{j}(0)$, $X_{j}(-1), \ldots, X_{j}\left(-\tau_{1}\right)$ by mathematical induction.

Proposition 3 Let $\Theta_{1}$ defined as the same as in Lemma 2, then we get that

$$
\ltimes_{j=1}^{M} X_{j}(t)=\Theta_{1}^{t} L_{t}^{\Theta_{1}} \ltimes_{j=1}^{M} X_{j}(0) \ltimes_{j=1}^{M} X_{j}(-1) \cdots \ltimes_{j=1}^{M} X_{j}\left(-\tau_{1}\right)
$$

where

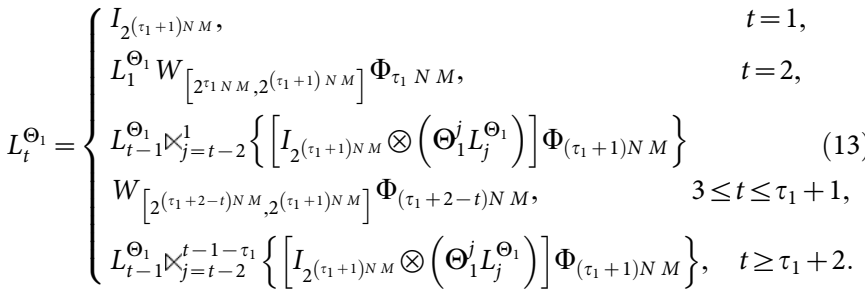

By Proposition 3, we then present another necessary and sufficient synchronization criterion for the array of TBNs.

Theorem 2 The array of TBNs can be synchronized iff there exists a positive integer $k$ such that

$\operatorname{Col}\left(\Theta_{1}^{k} L_{k}^{\Theta_{1}}\right) \subseteq\left\{\delta_{2^{M N}}^{\lambda_{i}}: \lambda_{i}=1+\frac{(i-1)\left(2^{M N}-1\right)}{2^{N}-1}, i=1,2, \ldots, 2^{N}\right\}$

where $L_{k}^{\Theta_{1}}$ is defined in Proposition 3.

Remark 5 According to equation (12), if we have an initial sequence, i.e. $\ltimes_{j=1}^{M} X_{j}(0) \ltimes_{j=1}^{M} X_{j}(-1) \cdots \ltimes_{j=1}^{M} X_{j}\left(-\tau_{1}\right)=\delta_{2^{N M\left(\tau_{1}+1\right)}}^{i}$, we can obtain that the value of states at time $k$ is $\operatorname{Col}_{i}\left[\Theta_{1}^{k} L_{k}^{\Theta_{1}}\right]$. Therefore, $\operatorname{Col}\left[\Theta_{1}^{k} L_{k}^{\Theta_{1}}\right]$ is the total possible state values at time t. In order to judge whether the coupled TBNs can be synchronized or not, we just need to know whether there exists a positive integer $k$ such that the possible state values are in the synchronous set $\left\{\delta_{2^{M N}}^{\lambda_{i}}: \lambda_{i}=1+\frac{(i-1)\left(2^{M N}-1\right)}{2^{N}-1}, i=1,2, \ldots, 2^{N}\right\}$.

Case II: $\boldsymbol{\tau}_{\mathbf{1}}<\boldsymbol{\tau}_{\mathbf{2}}$. Now we turn to case (II): $\tau_{1}<\tau_{2}$, where the initial state sequence is $X_{j}(0), X_{j}(-1), \ldots, X_{j}\left(-\tau_{2}\right)$ for $j=1,2, \ldots, M$. In this case, it means the state delay is smaller than the output delay. The following proposition is firstly presented to explicitly express the relationship between the current state and the initial state sequence.

Proposition 4 Let $\Theta_{2}=F W G \widehat{W}_{1}\left(I_{2^{\left(\tau_{2}+1\right) N M}} \otimes \widehat{W}_{2}\right) W_{\left[2^{\left(\tau_{1}+1\right) N M}, 2\left(\tau_{2}+1\right) N M\right.}$ $\Phi_{\left(\tau_{1}+1\right) N M}$ and $\Xi_{2}=\Theta_{2} W_{\left[2^{\tau_{2} N M}, 2\left(\tau_{2}+1\right) N M\right]}$, then we have

$\ltimes_{j=1}^{M} X_{j}(t+1)=\Theta_{2} \ltimes_{j=1}^{M} X_{j}(t) \ltimes_{j=1}^{M} X_{j}(t-1) \cdots \ltimes_{j=1}^{M} X_{j}\left(t-\tau_{2}\right)$

and

$\ltimes_{j=1}^{M} X_{j}(t)=\left(I_{2^{N M}} \otimes 1_{\tau_{2} N M}^{T}\right) \Xi_{2}^{t}\left\{\ltimes_{j=1}^{M} X_{j}(0) \ltimes_{j=1}^{M} X_{j}(-1) \cdots \ltimes_{j=1}^{M} X_{j}\left(-\tau_{2}\right)\right\}$.

In this case $\left(\tau_{1}<\tau_{2}\right)$, the initial state sequence is $X_{j}(0), \ldots, X_{j}\left(-\tau_{2}\right)$ for $j$ $=1,2, \ldots, M$. Thus, the STP of state of each TBN is determined by the initial state sequence and a transition matrix $\left(I_{2^{M N}} \otimes 1_{2^{\tau_{2} N M}}^{T}\right) \Xi_{2}^{t}$. Now we can present the following synchronization criterion which is similar with Theorem 1.

Theorem 3 Let equation (6) be the algebraic representations of the array of TBNs (1). Then synchronization occurs iff there exists a positive integer $k$ satisfying $1 \leq k \leq k_{0}$ such that

$\operatorname{Col}\left[\left(I_{2^{N M}} \otimes 1_{2^{\tau_{2} N M}}^{T}\right) \Xi_{2}^{k}\right] \subseteq\left\{\delta_{2^{M N}}^{\lambda_{i}}: \lambda_{i}=1+\frac{(i-1)\left(2^{M N}-1\right)}{2^{N}-1}, i=1,2, \ldots, 2^{N}\right\}$

where 


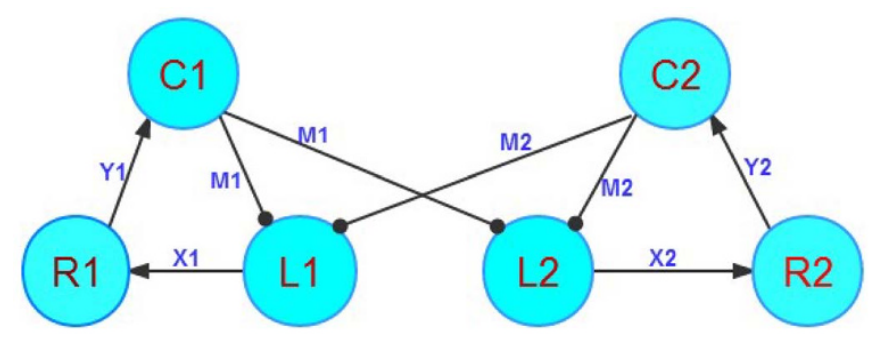

Figure 1 Boolean model of a coupled macromolecular synthesis in a cell with each unit consisting of a genetic locus $\left(L_{1}\right.$ or $\left.L_{2}\right)$, a cellular structure $\left(R_{1}\right.$ or $R_{2}$ ) and a cellular locus $\left(C_{1}\right.$ or $\left.C_{2}\right)$. Arrows correspond to positive interactions, circles to negative ones.

$$
k_{0}=\min \left\{i: i \geq 1,\left(I_{2^{N M}} \otimes 1_{2^{\tau_{2} N M}}^{T}\right) \Xi_{2}^{i}=\left(I_{2^{N M}} \otimes 1_{2^{\tau_{2} N M}}^{T}\right) \Xi_{2}^{j} \text { for some } j>i\right\} .
$$

Similar to Theorem 1, we can also obtain the same synchronous set, which is $\quad\left\{\delta_{2^{M N}}^{\lambda_{i}}: \lambda_{i}=1+\frac{(i-1)\left(2^{M N}-1\right)}{2^{N}-1}, i=1,2, \ldots, 2^{N}\right\}$. According to equation (16), we can obtain the total possible state values as $\operatorname{Col}\left[\left(I_{2^{N M}} \otimes 1_{2^{\tau_{2} N M}}^{T}\right) \Xi_{2}^{k}\right]$. As long as there exists a positive integer $k$ such that the possible state values at time $k$ are in the synchronous set, then we can draw a conclusion that the coupled TBNs can be synchronized.

Remark 6 In equation (18) in Theorem 3, we can also use $k_{0}^{\prime}=\min \left\{i \geq 1: \Xi_{2}^{i}=\Xi_{2}^{j}, j>i\right\}$ to simplify it but meanwhile the restriction is strengthened. And we can also get $k_{0} \leq k_{0}^{\prime}$. Equation (17) implies that after finite time steps, i.e. $k_{0}$ steps, we can judge whether the array of TBNs can be synchronized or not. If after $k_{0}$ steps, this array of TBNs still can not be synchronized, then it can not be synchronized at any time. Thus, Theorem 3 also provides an efficient criterion for synchronization in an array of output-coupled TBNs.

Since $\left(I_{2^{N M}} \otimes 1_{2^{\tau_{2} N M}}^{T}\right)$ and $\Xi_{2}$ are logical matrices, we can derive the following simplified criterion for synchronization in an array of output-coupled TBNs.

Corollary 2 Let $\Omega_{2}=\left\{\left(\lambda_{i}-1\right) 2^{\tau_{2} N M}+j: \lambda_{i}=1+\frac{(i-1)\left(2^{N M}-1\right)}{2^{N}-1}\right.$, $\left.i=1,2, \ldots, 2^{N}, j=1, \ldots, 2^{\tau_{1} N M}\right\}$. Then the array of temporal Boolean networks can be synchronized iff there exists a positive integer $k$ such that $\operatorname{Row}_{i}\left(\Xi_{2}^{k}\right)=\underbrace{(0,0, \ldots, 0)}_{2^{\left(\tau_{2}+1\right) N M}}, i \in\left\{1,2, \ldots, 2^{\left(\tau_{2}+1\right) N M}\right\} / \Omega_{2}$.

Similar to Proposition 3, we can also use mathematical induction to obtain following proposition which implies the relationship between the current state and the initial state sequence by using Proposition 4.

Proposition 5 Let $\Theta_{2}$ be defined the same as in Proposition 4, then we can obtain that

$$
\ltimes_{j=1}^{M} X_{j}(t)=\Theta_{2}^{t} L_{t}^{\Theta_{2}} \ltimes_{j=1}^{M} X_{j}(0) \ltimes_{j=1}^{M} X_{j}(-1) \ldots \times_{j=1}^{M} X_{j}\left(-\tau_{2}\right)
$$

\begin{tabular}{|c|c|}
\hline variable & Boolean function \\
\hline $\begin{array}{l}x_{1}^{1}=\text { genetic locus } L_{1} \\
x_{1}^{2}=\text { ribosome } R_{1} \\
x_{1}^{3}=\text { cellular locus } C_{1} \\
x_{2}^{1}=\text { genetic locus } L_{2} \\
x_{2}^{2}=\text { ribosome } R_{2} \\
x_{2}^{3}=\text { cellular locus } C_{2}\end{array}$ & $\begin{array}{c}\neg x_{1}^{3} \wedge y_{2} \\
x_{1}^{1} \\
x_{1}^{2} \\
\neg x_{2}^{3} \wedge y_{1} \\
x_{2}^{1} \\
x_{1}^{2}\end{array}$ \\
\hline
\end{tabular}

where

$$
L_{t}^{\Theta_{2}}=\left\{\begin{array}{lc}
I_{2^{\left(\tau_{2}+1\right) N M}}, & t=1, \\
\left.L_{1}^{\Theta_{2}} W_{\left[2^{\tau_{2} N M, 2}\right.}{ }^{\left(\tau_{2}+1\right) N M}\right] \Phi_{\tau_{2} N M}, & t=2, \\
L_{t-1}^{\Theta_{2}} \ltimes_{j=t-2}^{1}\left\{\left[I_{2^{\left(\tau_{2}+1\right) N M}} \otimes\left(\Theta_{2}^{j} L_{j}^{\Theta_{2}}\right)\right] \Phi_{\left(\tau_{2}+1\right) N M}\right\} & (20) \\
W_{\left[2^{\left(\tau_{2}+2-t\right) N M}, 2^{\left(\tau_{2}+1\right) N M}\right]} \Phi_{\left(\tau_{2}+2-t\right) N M}, & 3 \leq t \leq \tau_{2}+1, \\
L_{t-1}^{\Theta_{2}} \ltimes_{j=t-2}^{t-1-\tau_{2}}\left\{\left[I_{2^{\left(\tau_{2}+1\right) N M}} \otimes\left(\Theta_{2}^{j} L_{j}^{\Theta_{2}}\right)\right] \Phi_{\left(\tau_{2}+1\right) N M}\right\}, t \geq \tau_{2}+2 .
\end{array}\right.
$$

According to Proposition 5, we can obtain following synchronization criterion to judge the array of output-coupled TBNs can be synchronized or not, which is similar to Theorem 2.

Theorem 4 The array of TBNs can be synchronized iff there exists a positive integer $k$ such that

$\operatorname{Col}\left(\Theta_{2}^{k} L_{k}^{\Theta_{2}}\right) \subseteq\left\{\delta_{2^{M N}}^{\lambda_{i}}: \lambda_{i}=1+\frac{(i-1)\left(2^{M N}-1\right)}{2^{N}-1}, i=1,2, \ldots, 2^{N}\right\}$

where $L_{k}^{\Theta_{2}}$ is defined in Proposition 5.

According to equation (19), we obtain the total possible state values are $\operatorname{Col}\left(\Theta_{2}^{k} L_{k}^{\Theta_{2}}\right)$. Following similar discussions in Remark 5 , the coupled TBNs can be synchronized if and only if there exists an integer $k$ such that the possible state values are in the synchronization set.

\section{Numerical Simulation}

In this section, we present two numerical examples to illustrate our main theoretical results, which include a practical epigenetic example.

Example 1 Let us first consider the BN which is used to model a molecular biological system ${ }^{41}$. It is an epigenetic model proposed by Goodwin ${ }^{42}$ of control mechanisms for the macromolecular synthesis in cells including: genetic locus $L_{i}$, cellular structure $R_{i}$ (ribosome) and cellular locus $C_{i}, i=1,2$. As is shown in Fig. $1, L_{i}$ synthesises $m R N A$ $\left(X_{i}\right)$ to the cellular structure $R_{i}$, where its activity results in the synthesis of a protein, denoted by $Y_{i}$. The protein then travels to some cellular locus $C_{i}$. A fraction of a metabolic species $M_{i}$ generated by the protein returns to the genetic locus $L_{i}$. Moreover, a reciprocal interaction occurs from $L_{2}$ to $L_{1}$. Based on the analysis $i^{41}$, the variables and Boolean functions of the model are given in Table $I$.

Let $f_{1}=\left(f_{1}^{1}, f_{1}^{2}, f_{1}^{3}\right)=\left(\neg x_{1}^{3} \wedge y_{2}, x_{1}^{1}, x_{1}^{2}\right), f_{2}=\left(f_{2}^{1}, f_{2}^{2}, f_{2}^{3}\right)=\left(\neg x_{2}^{2} \wedge y_{1}, x_{2}^{1}, x_{2}^{2}\right)$, $g_{1}=\neg x_{1}^{3}, g_{2}=\neg x_{2}^{3}$. During the process of synthesis in cells, several environmental conditions such as temperature, growth rate or concentration of nutrition, may cause a time delay of state and output. Thus, it is reasonable to use TBNs in form (1) to model the network. Meanwhile, we can use the following case to illustrate the impacts of time delay occurred in the process of synthesis in cells towards the synchronization problem. Put $x_{j}(t)=x_{j}^{1}(t) \ltimes x_{j}^{2}(t) \ltimes x_{j}^{3}(t)$ for $j=1,2$, and $y(t)=y_{1}(t) \ltimes y_{2}(t)$. In the $T B N s$, we let the initial state sequences be $x_{1}(-1)=(1,1,1), x_{2}(-1)=(0$, $0,0), x_{1}(0)=(0,0,0), x_{2}(0)=(1,1,1)$.

Consider: $\tau_{1}=\tau_{2}=1$ which means that the state time delay is equal to the output time delay. 
(a1)
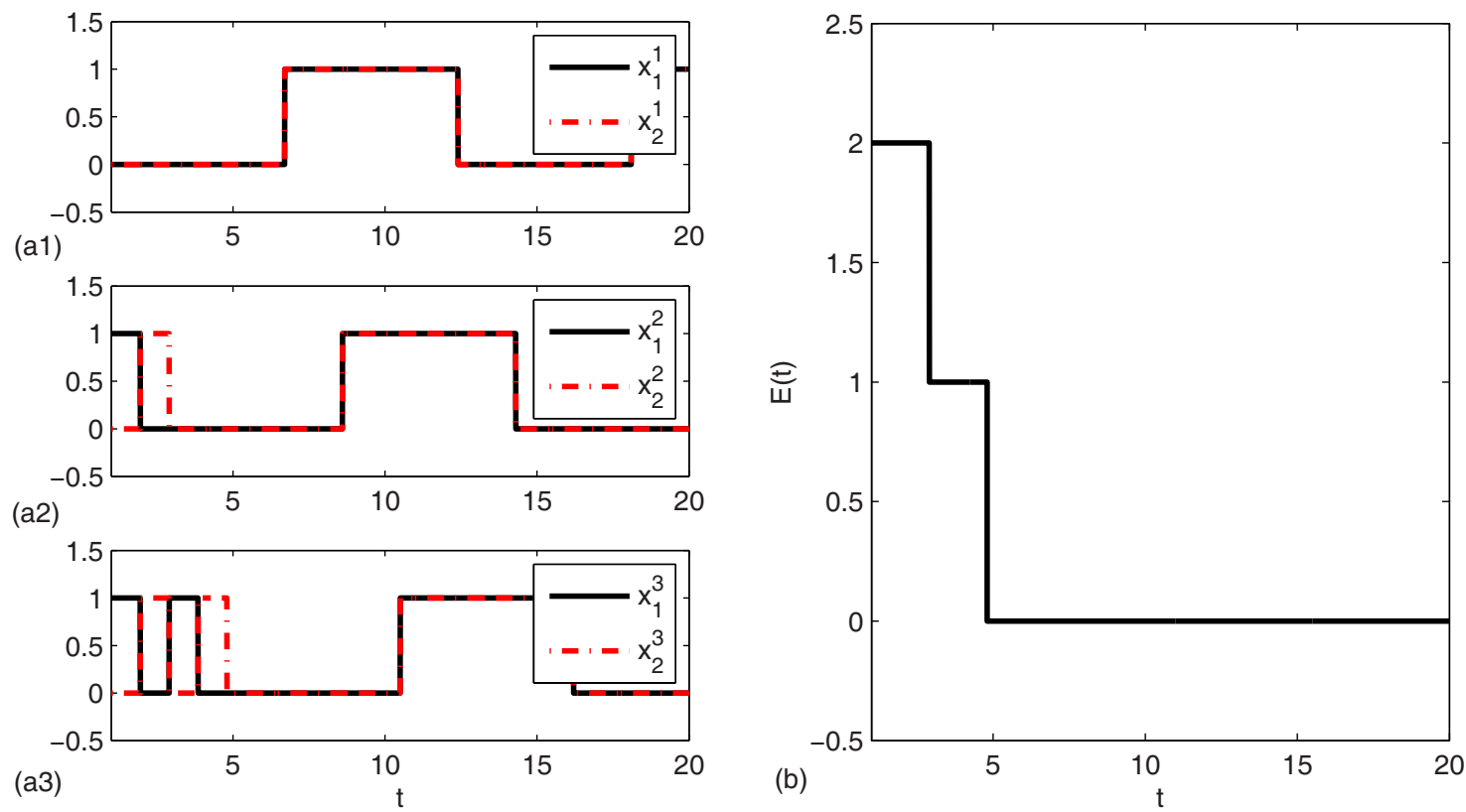

(a3)

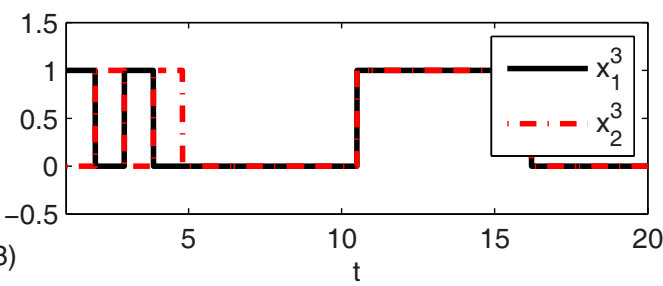

(b)

Figure $2 \mid$ (a): Time evolution of TBNs (22) with the given initial state sequences. The black solid line presents the state evolution of $x_{1}$, while the red dash line presents the state evolution of $x_{2}$. (b): Total synchronization error $E(t)$ of the coupled BNs (22) with the given initial state sequences.

By using the STP of matrices, we also obtain the following algebraic representation:

$$
\left\{\begin{array}{l}
x_{1}(t+1)=F x_{1}(t-1) y_{2}(t) \\
x_{2}(t+1)=F x_{2}(t-1) y_{1}(t) \\
y_{1}(t)=G x_{1}(t-1) \\
y_{2}(t)=G x_{2}(t-1)
\end{array}\right.
$$

where $\mathrm{F}=\delta_{8}[5,5,1,5,6,6,2,6,7,7,3,7,8,8,4,8]$ and $G=\delta_{8}[2,1,2$, $1,2,1,2,1]$.

In order to see whether these two TBNs can be synchronized or not, we should calculate $\Xi_{1}^{t}$ according to Theorem 1. Direct computation gives that $\Xi_{1}^{5}=\Xi_{1}^{17}$, then we have $k_{0}^{\prime}=5$. According to Remark 3, we have $k_{0} \leq k_{0}^{\prime}=5$. Moreover, we obtain that $\left(I_{2^{6}} \otimes 1_{2^{6}}^{T}\right) \Xi_{1}^{5} \subseteq$ $\left\{\delta_{64}^{i} \mid i=1,10,19,28,37,46,55,64\right\}$. Hence, the TBNs can be synchro-

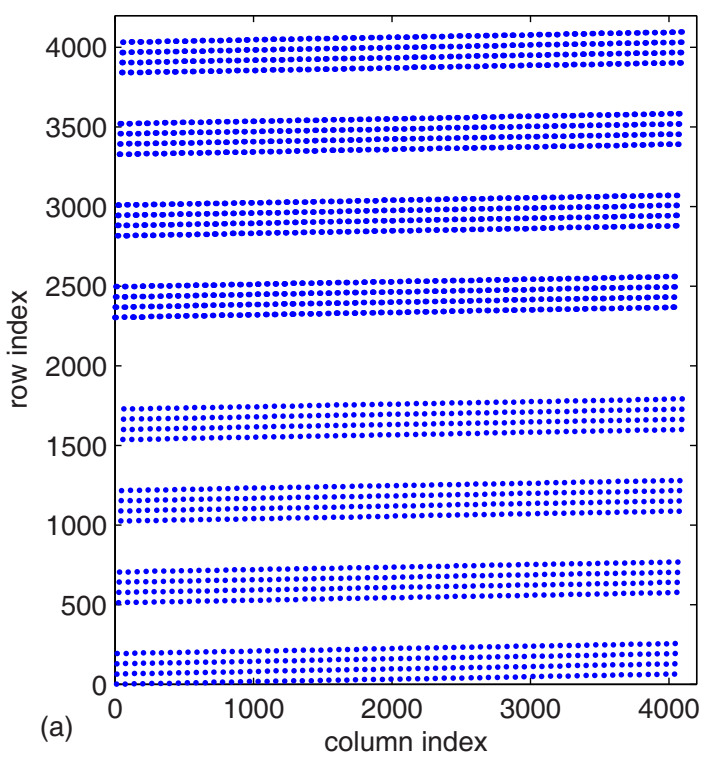

nized by Theorem 1. Define the total synchronization error: $E(t)=\sum_{i=1}^{3}\left|x_{1}^{i}(t)-x_{2}^{i}(t)\right|$. Fig. 2(a) shows the time evolution of the TBNs and Fig. 2(b) shows the total synchronization error $E(t)$. By Proposition 2, we also get $\Xi_{1}$. Due to the complexity of this matrix, we just present the indexes of the first 50 columns as follows:

$\Xi_{1}=\delta_{2^{12}}[2305,2305,2369,2369,2433,2433,2497,2497$,

65, 2433, 129, 2497, 193, 2817, 2817, 2881, 2881,

2945, 2945, 3009, 3009, 2817, 513, 2881, 577, 2945,

641, 3009, 705, 3329, 3329, 3393, 3393, 3457, 3457,

$3521,3521,3329,1025,3393,1089,3457,1153$,

$3521,1217,3841,3841, \ldots]$

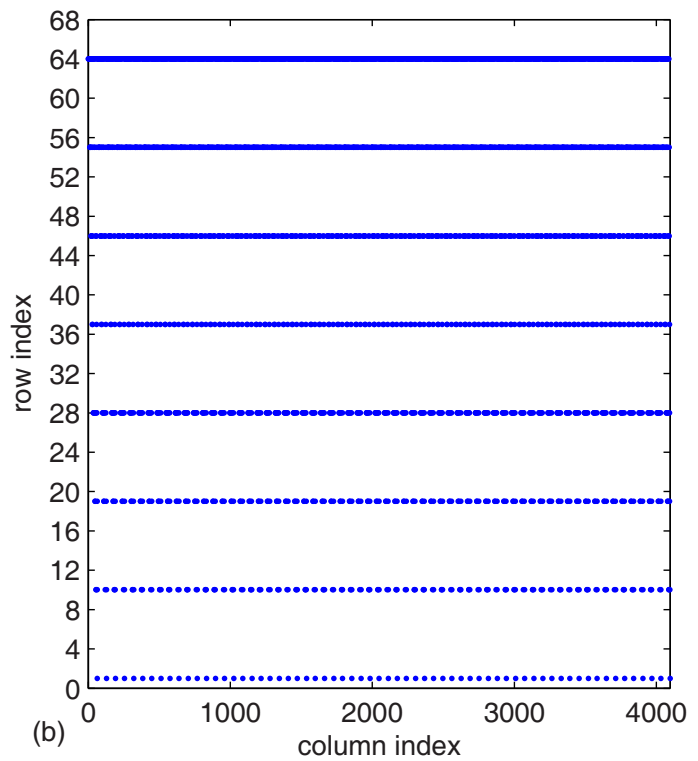

Figure $3 \mid$ (a): The whole row indexes of each column of matrix $\Xi_{1}$. (b): The whole row indexes of each column of matrix $\left(I_{2^{6}} \otimes \mathbf{1}_{2^{6}}^{T}\right) \Xi_{1}^{5}$. Each point corresponds to the row index of each column, which implies the position of 1 . 


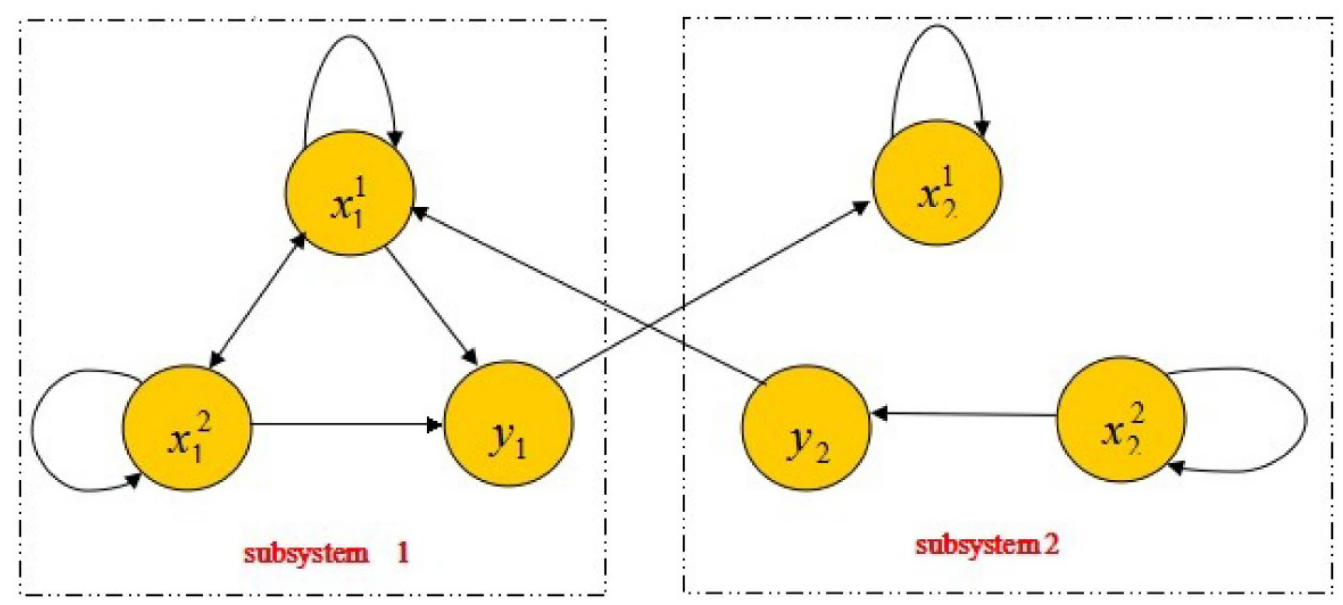

Figure $4 \mid$ Two coupled BNs with each network consisting of two nodes and one output. Arrows correspond to interactions between nodes and outputs.

and Fig. 3(a) shows the whole row indexes of each column of matrix $\Xi_{1}$, Fig. 3(b) shows the row indexes of each column of matrix $\left(I_{2^{6}} \otimes 1_{2^{6}}^{T}\right) \Xi_{1^{5}}^{5}$.

Example 2 In Fig. 4, we have a system consisting of two coupled TBNs with delay $\tau_{1}=\tau_{2}=1$, each TBN with two nodes. And its dynamics is described as follows:

$$
\begin{aligned}
& \left\{\begin{array}{l}
x_{1}^{1}(t+1)=\left[x_{1}^{2}(t) \wedge x_{1}^{1}(t)\right] \rightarrow y_{2}(t), \\
x_{1}^{2}(t+1)=\left[x_{1}^{1}(t) \leftrightarrow x_{1}^{2}(t)\right], \\
y_{1}(t)=x_{1}^{1}(t) \vee x_{1}^{2}(t-1) .
\end{array}\right. \\
& \left\{\begin{array}{l}
x_{2}^{1}(t+1)=x_{2}^{1}(t-1) \leftrightarrow y_{1}(t), \\
x_{2}^{2}(t+1)=x_{2}^{2}(t) \wedge x_{2}^{2}(t-1), \\
y_{2}(t)=x_{2}^{2}(t-1)
\end{array}\right.
\end{aligned}
$$

Denote $x_{j}(t)=x_{j}^{1}(t) \ltimes x_{j}^{2}(t)$ for $j=1,2$ and $y(t)=y_{1}(t) \ltimes y_{2}(t)$, then we can express the system into its algebraic form by using the STP of matrices as follows:

$$
\left\{\begin{aligned}
x_{1}^{1}(t+1) & =F_{1}^{1} x_{1}(t) x_{1}(t-1) y(t) \\
x_{1}^{2}(t+1) & =F_{1}^{2} x_{1}(t) x_{1}(t-1) y(t) \\
y_{1}(t) & =G_{1} x_{1}(t) x_{1}(t-1)
\end{aligned}\right.
$$

and

$$
\left\{\begin{aligned}
x_{2}^{1}(t+1) & =F_{2}^{1} x_{2}(t) x_{2}(t-1) y(t) \\
x_{2}^{2}(t+1) & =F_{2}^{2} x_{2}(t) x_{2}(t-1) y(t) \\
y_{2}(t) & =G_{2} x_{2}(t) x_{2}(t-1)
\end{aligned}\right.
$$

We obtain the explicit expression of the matrices $F_{1}^{1}, F_{1}^{2}, F_{2}^{1}, F_{2}^{2}, G_{1}$ and $G_{2}$, by using STP. Here, we omit the procedure of computation for simplicity.

As we can see this system belongs to case (I). By Proposition 2, we obtain the matrix $\Xi_{1}$ and Fig. 5 shows the row indexes of each columns of matrix $\Xi_{1}$. In order to judge whether these two coupled TBNs can be synchronized or not, we should calculate $\Xi_{1}^{t}$ according to Theorem 1 . Direct computation shows that $\left(I_{2^{4}} \otimes 1_{2^{4}}^{T}\right) \Xi_{1}^{8}=\left(I_{2^{4}} \otimes I_{2^{4}}^{T}\right) \Xi_{1}^{13}$ by using

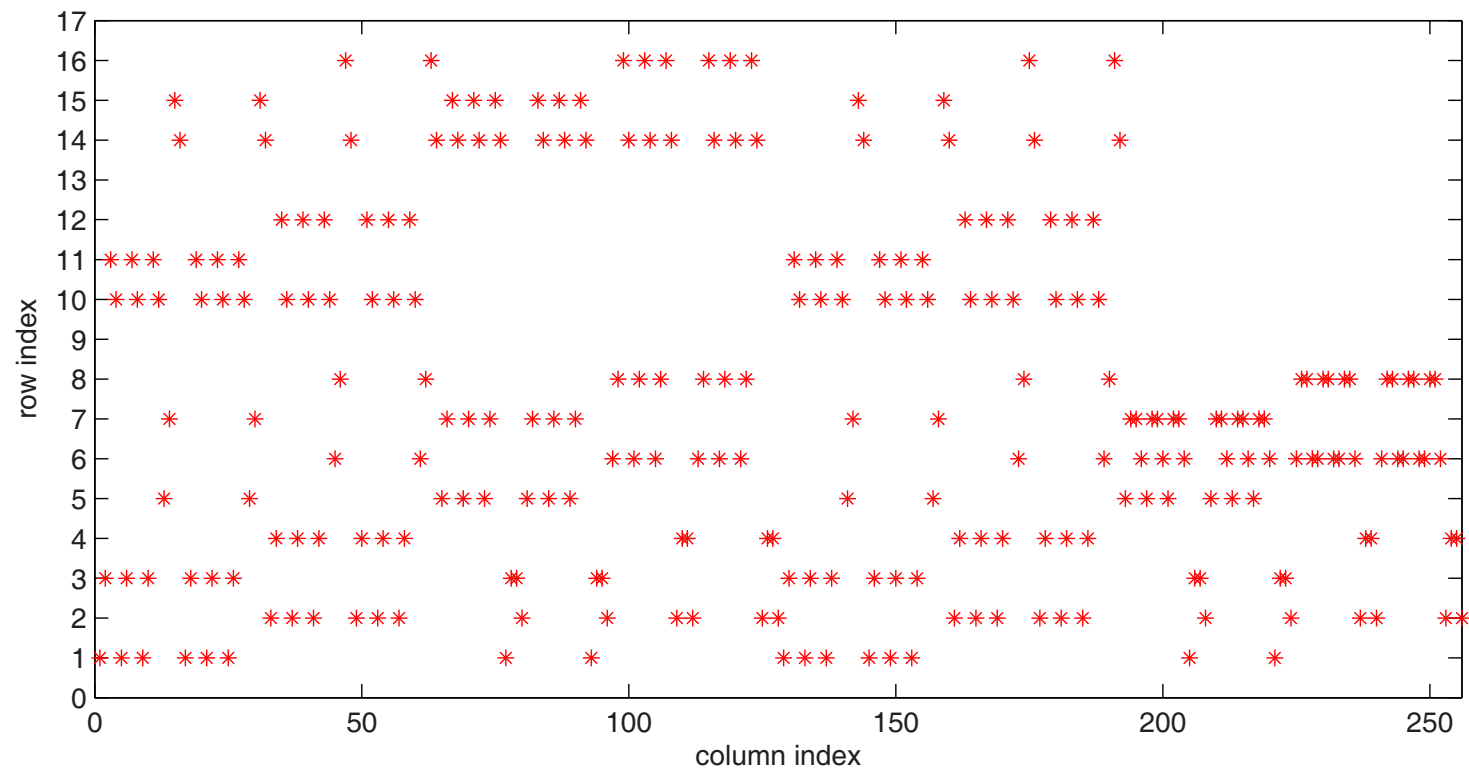

Figure $5 \mid$ The whole row indexes of each column of matrix $\Xi_{1}$ obtained in system (24). Each point corresponds to the row index of each column, which implies the position of 1 . 

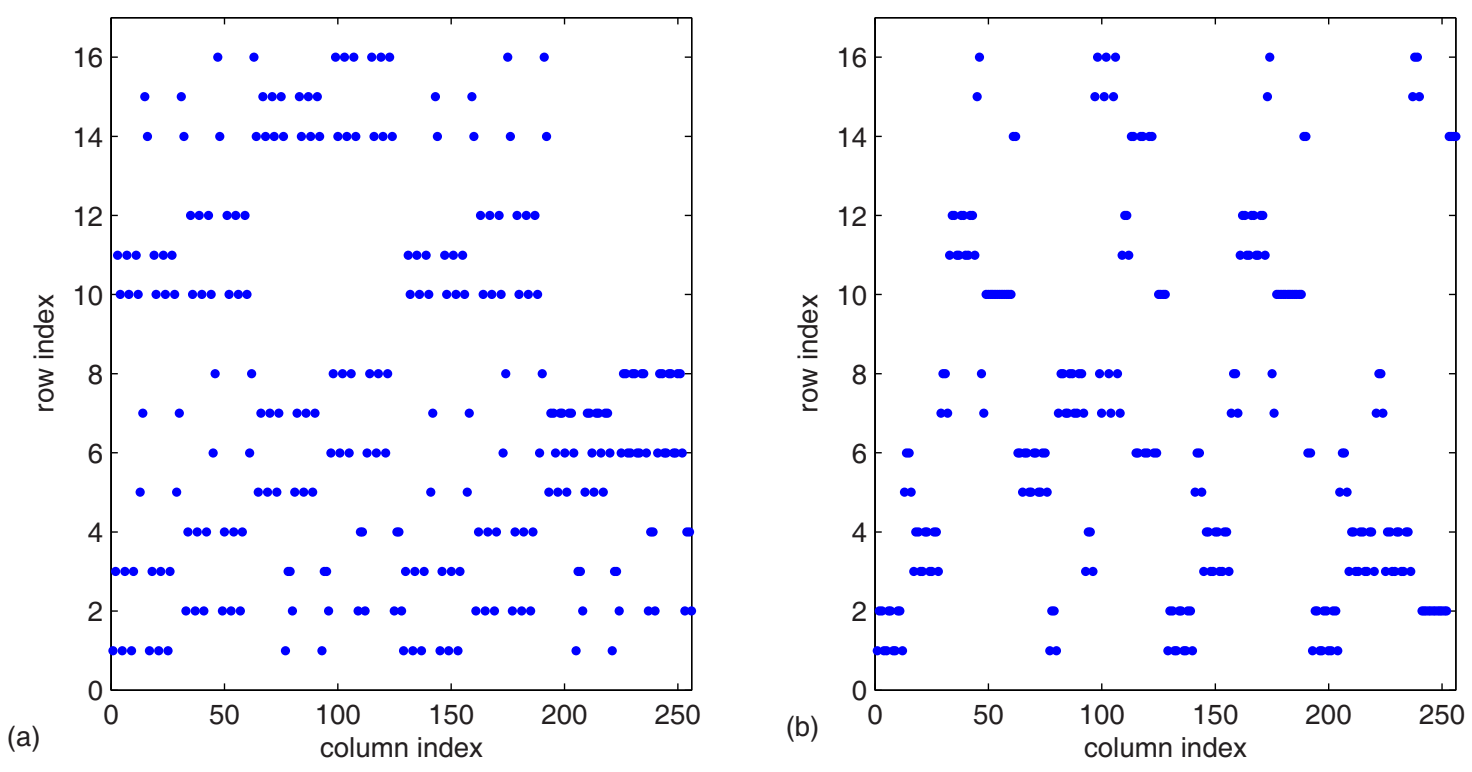

Figure $6 \mid$ (a): The whole row index of each column of matrix $\left(I_{2^{4}} \otimes \mathbf{1}_{2^{4}}^{T}\right) \Xi_{1}$ obtained in system (24). (b): The whole row index of each column of matrix $\left(I_{2^{4}} \otimes \mathbf{1}_{2^{4}}^{T}\right) \Xi_{1}^{2}$. Each point corresponds to the row index of each column, which implies the position of 1 .

the equation (11), then we have $k_{0}=8$. However, we get that $\Xi_{1}^{9}=\Xi_{1}^{14}$, while $\Xi_{1}^{8} \neq \Xi_{1}^{13}$. By using $k_{0}^{\prime}=\min \left\{i \geq 1: \Xi_{1}^{i}=\Xi_{1}^{j}, j>i\right\}$, we have $k_{0}^{\prime}=9$ which verifies Remark 3 . It is easy to verify that $\operatorname{Col}\left[\left(I_{2^{4}} \otimes 1_{2^{4}}^{T}\right) \Xi_{1}^{t}\right] \nsubseteq\left\{\delta_{2^{4}}^{i}: i=1,6,11,16\right\}$ for $1 \leq t \leq 8$. In Fig. 6 to Fig. 9, we plot the whole row index of columns of each matrix $\left(I_{2^{4}} \otimes 1_{2^{4}}^{T}\right) \Xi_{1}^{i}, i=1,2, \ldots, 8$. From these figures, we can also draw the conclusion that $\operatorname{Col}\left[\left(I_{2^{4}} \otimes 1_{2^{4}}^{T}\right) \Xi_{1}^{t}\right] \nsubseteq\left\{\delta_{2^{4}}^{i}: i=1,6,11,16\right\}$ for $1 \leq$ $t \leq 8$. Hence, this output-coupled TBNs can not be synchronized according to Theorem 1 .

In the following, we can also define the total synchronization error of the output-coupled TBNs: $E(t)=\left|x_{1}^{1}(t)-x_{2}^{1}(t)\right|+\left|x_{1}^{2}(t)-x_{2}^{2}(t)\right|$. Fig. 10(a) shows the time evolution of the state $x_{1}(t)$ and $x_{2}(t)$ of the array of TBNs (24), and Fig. 10(b) shows the totaly synchronization error $E(t)$ of the TBNs (24). From the state evolution and the total synchronization error of the TBNs (24), we can draw the conclusion that the TBNs can not synchronized. Our analytical results are well illustrated by this example.

Remark 7 Due to the complexity of computation, Fig. 10(a) and Fig. 10(b) just plot time evolution and the total synchronization error of the TBNs with time up to $t=100$. To some extent, Fig. 10(a) and Fig. 10(b) can illustrate that the coupled TBNs can not be synchronized. In fact, we have already made the computation of these TBNs with time up to $t=10000$, observing that these TBNs can not be synchronized. Since the simulation result can not be well expressed in the figures with time $t=10000$, we just plot Fig. 10(a) and Fig. 10(b) till time $t=100$ only.

\section{Conclusions}

In this paper, we have studied synchronization in an array of outputcoupled TBNs. Both a state delay $\tau_{1}$ and an output delay $\tau_{2}$ are considered in the model. In a genetic regulatory network, state corre-
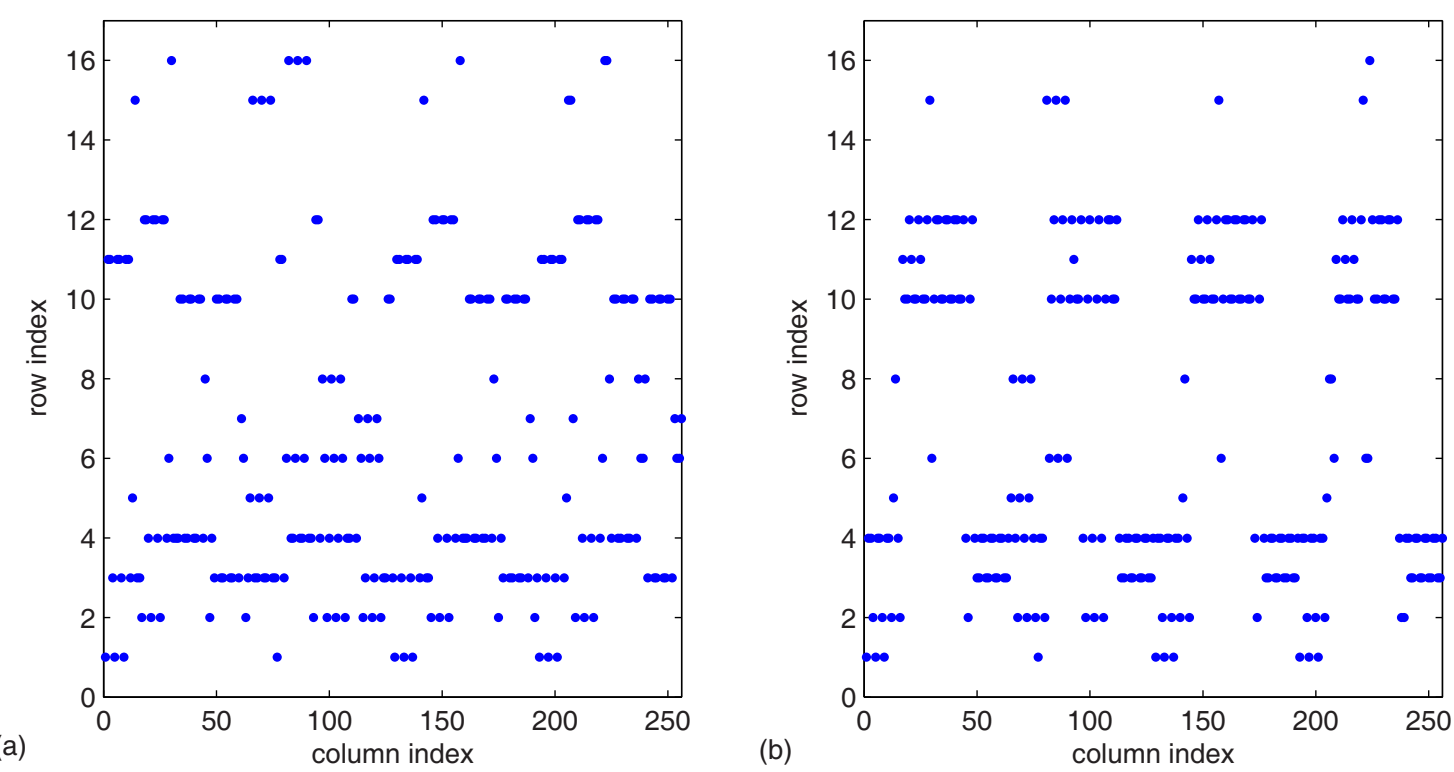

Figure $7 \mid$ (a): The whole row index of each column of matrix $\left(I_{2^{4}} \otimes \mathbf{1}_{2^{4}}^{T}\right) \Xi_{1}^{3}$ obtained in system (24). (b): The whole row index of each column of matrix $\left(I_{2^{4}} \otimes \mathbf{1}_{2^{4}}^{T}\right) \Xi_{1}^{4}$. Each point corresponds to the row index of each column, which implies the position of 1 . 
(a)

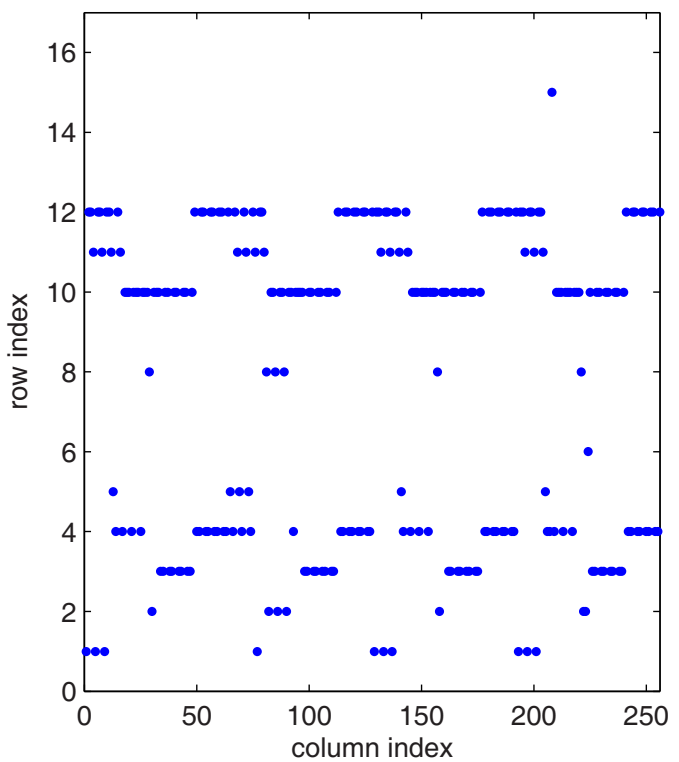

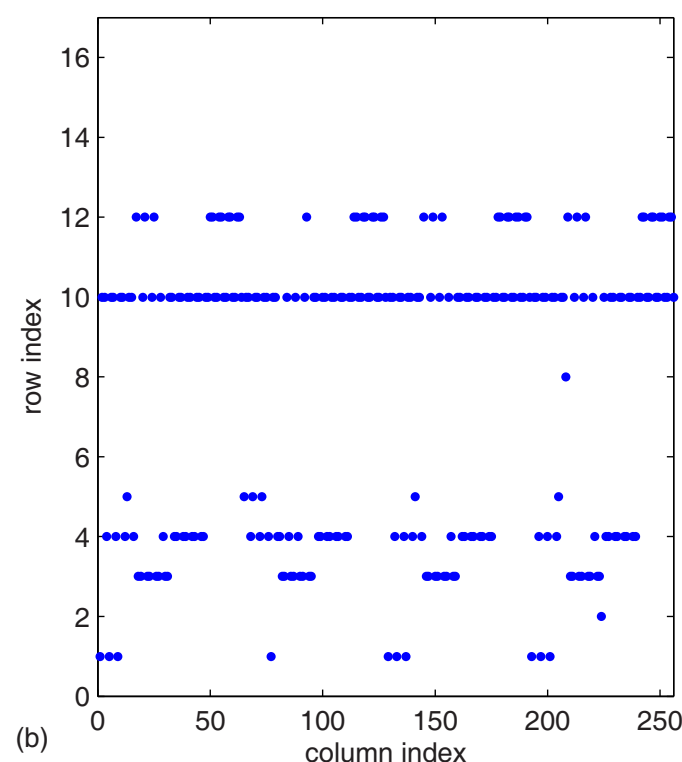

Figure $8 \mid$ (a): The whole row index of each column of matrix $\left(I_{2^{4}} \otimes \mathbf{1}_{2^{4}}^{T}\right) \Xi_{1}^{5}$ obtained in system (24). (b): The whole row index of each column of matrix $\left(I_{2^{4}} \otimes \mathbf{1}_{2^{4}}^{T}\right) \Xi_{1}^{6}$. Each point corresponds to the row index of each column, which implies the position of 1 .

sponds to the level of gene, while the output corresponds to gene products. In real world, many evolutionary processes may experience external or internal changes. These changes maybe due to the environmental disturbances or the interconnections of other genes, which can heavily affect the evolutional processes of genes, such as the transcription process or the translation process. By using the semitensor product of matrices, we first convert the TBNs into an unique discrete time algebraic system. Based on the equivalent algebraic representations of TBNs, we then study the coupled TBNs by considering the two cases: $\tau_{1} \geq \tau_{2}$ and $\tau_{1}<\tau_{2}$. For each case, we derive necessary and sufficient synchronization criteria for the array of output-coupled TBNs. Different types of criteria are derived from different aspects. These criteria provide efficient ways to judge whether an array of output-coupled TBNs can be synchronized or not. And they also implies that if the array of TBNs can not be synchronized among the upper bound time, then they can not be synchronized at any time, which means we only need to judge finite time steps. Finally, two illustrative examples including a practical epigenetic example are given to show the validity of our theoretical results. One interesting topic in the near future is to design logical controllers to make coupled TBNs synchronized.

This mechanism is expected to be applicable to a wide range of biological systems, because synchronization phenomenon is very commonly in biological systems. Moreover, it is expected to help to understand the nature of cellular function, the behavior of genes and the manner in which cells execute and control a large number of operations which are required for normal function and the ways in which cellular systems fail in disease.
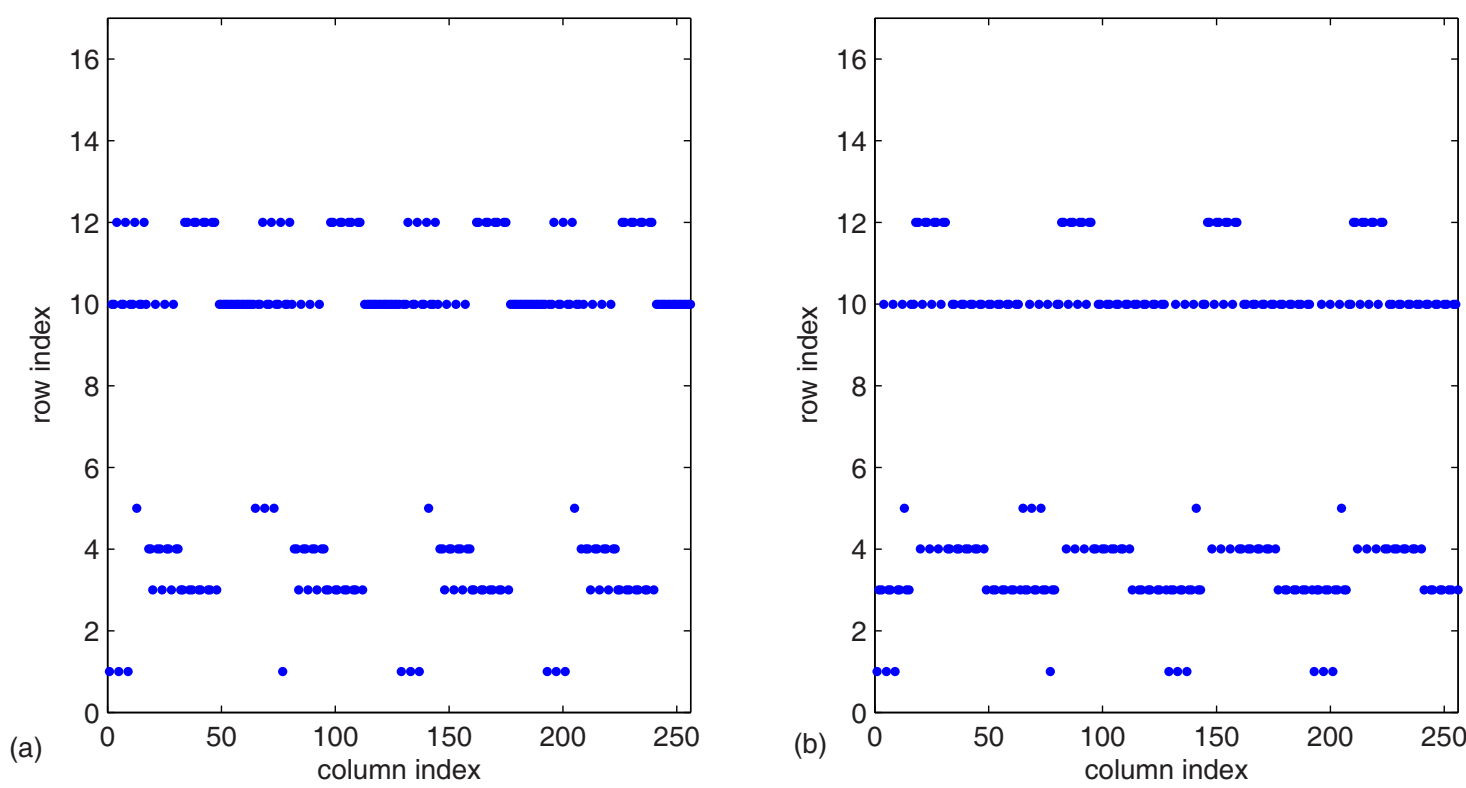

Figure $9 \mid$ (a): The whole row index of each column of matrix $\left(I_{2^{4}} \otimes \mathbf{1}_{2^{4}}^{T}\right) \Xi_{1}^{5}$ obtained in system (24). (b): The whole row index of each column of matrix $\left(I_{2^{4}} \otimes \mathbf{1}_{2^{4}}^{T}\right) \Xi_{1}^{6}$. Each point corresponds to the row index of each column, which implies the position of 1 . 

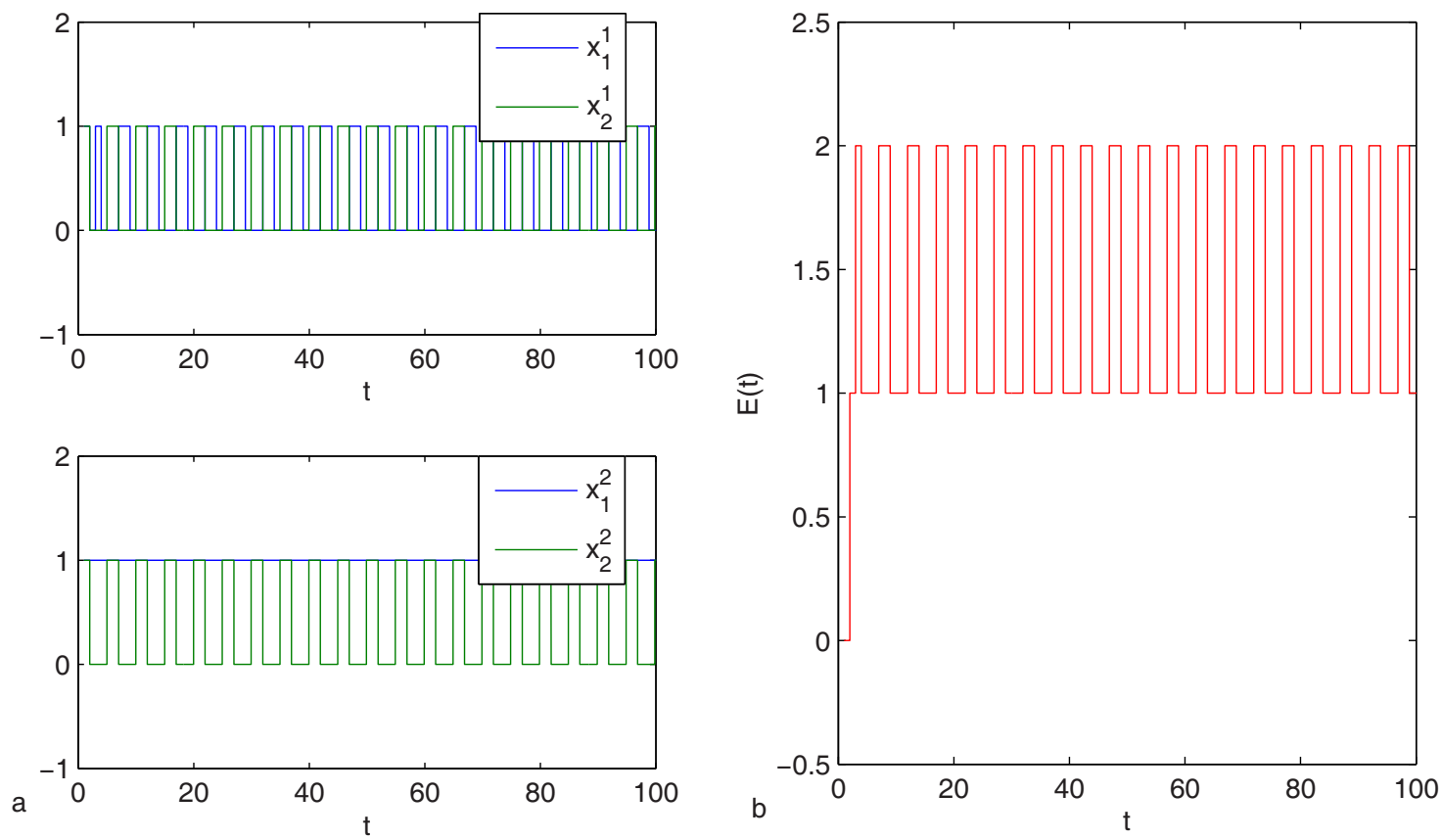

Figure $10 \mid$ (a): Time evolution of the TBNs $(24)$ with initial state sequences $x_{1}(-1)=(0,0), x_{2}(-1)=(1,1), x_{1}(0)=(1,0), x_{2}(0)=(0,1)$. The red solid line
presents the state evolution of $x_{1}$, while the green solid line presents the state evolution of $x_{2}$. (b): Total synchronization error $E(t)$ of the TBNs $(24)$. Fig. (a) shows states $x_{1}^{1}$ and $x_{2}^{1}$, states $x_{1}^{2}$ and $x_{2}^{2}$ can not reach synchronization till time to 100, while Fig. (b) shows the total synchronization error fluctuates between value 2 and value 0 . Both Fig. (a) and Fig. (b) imply that the coupled TBNs can not be synchronized.

1. Kauffman, S. A. Metabolic stability and epigenesis in randomly constructed genetic nets. J. Theor. Biol. 22, 437-467 (1969).

2. Kim, J., Park, S. M. \& Cho, K. H. Discovery of a kernel for controlling biomolecular regulatory networks. Sci. Rep. 3, 2223 (2013).

3. Huang, S. \& Ingber, D. E. Shape-dependent control of cell growth, differentiation, and apoptosis: switching between attractors in cell regulatory networks. Exp. Cell Res. 261, 91-103 (2000).

4. Kürten, K. Correspondence between neural threshold networks and Kauffman Boolean cellular automata. J. Phys. A: Math. Gen. 21, L615-L619 (1988).

5. Huang, C., Ho, D. W. C. \& Lu, J. Q. Partial-information-based distributed filtering in two-targets tracking sensor network. IEEE Trans. Circuits Syst. I, Reg. Papers 59, 820-832 (2012).

6. Shmulevich, I., Lähdesmäki, H., Dougherty, E. R., Astola, J. \& Zhang, W. The role of certain Post classes in Boolean network models of genetic networks. Proc. Natl. Acad. Sci. USA 100, 10734-10739 (2003).

7. Li, L. L., Ho, D. W. C. \& Lu, J. Q. A unified approach to practical consensus with quantized data and time delay. IEEE Trans. Circuits Syst. I, Reg. Papers 60 2668-2678 (2013).

8. Lu, J. Q., Cao, J. D. \& Ho, D. W. C. Adaptive stabilization and synchronization for chaotic Lur'e systems with time-varying delay. IEEE Trans. Circuits Syst. I, Reg. Papers 55, 1347-1356 (2008).

9. Li, F. T., Long, T., Lu, Y., Ouyang, Q. \& Tang, C. The yeast cell-cycle network is robustly designed. Proc. Natl. Acad. Sci. USA 101, 4781-4786 (2004).

10. Faure, A., Naldi, A., Chaouiya, C. \& Thieffry, D. Dynamical analysis of a generic Boolean model for the control of the mammalian cell cycle. Bioinform. 22, e124-e131 (2006).

11. Kauffman, S. A., Peterson, C., Samulesson, B. \& Troein, C. Random Boolean network models and the yeast transcriptional network. Proc. Natl. Acad. Sci. USA 100, 14796-14799 (2003).

12. Albert, R. \& Othmer, H. G. The topology of the regulatory interactions predicts the expression pattern of the segment polarity genes in Drosophila melanogaster. J. Theor. Biol. 223, 1-18 (2003).

13. Cheng, D. Z. \& Qi, H. S. A linear representation of dynamics of Boolean networks. IEEE Trans. Autom. Control 55, 2251-2258 (2010).

14. Cheng, D. Z., Qi, H. S. \& Li, Z. Q. Analysis and Control of Boolean Networks: A Semi-tensor Product Approach. (Springer, 2011).

15. Li, F. F. \& Sun, J. T. Stability and stabilization of Boolean networks with impulsive effects. Syst. Control Lett. 61, 1-5 (2012).

16. Zhao, Y., Li, Z. Q. \& Cheng, D. Z. Optimal control of logical control networks. IEEE Trans. Autom. Control 56, 1766-1776 (2011).

17. Arenas, A., Díaz-Guilera, A., Kurths, J., Moreno, Y. \& Zhou, C. S. Synchronization in complex networks. Phys. Rep. 469, 93-153 (2008).

18. Tang, Y., Gao, H. J. \& Kurths, J. Multiobjective identification of controlling areas in neuronal networks. IEEE/ACM Trans. Comput. Biol. Bioinform. 10, 708-720 (2013).
19. Baptista, M. S., Ren, H. P., Swarts, J. C. M., Carareto, R., Nijmeijer, H. \& Grebogi, C. Collective almost synchronisation in complex networks. PloS ONE 7, e48118 (2012).

20. Lu, J. Q., Kurths, J., Cao, J. D., Mahdavi, N. \& Huang, C. Synchronization control for nonlinear stochastic dynamical networks: pinning impulsive strategy. IEEE Trans. Neural Netw. Lear. Syst. 23, 285-292 (2012).

21. Song, Q. \& Cao, J. D. On pinning synchronization of directed and undirected complex dynamical networks. IEEE Trans. Circuits Syst. I, Reg. Papers 57, 672-680 (2010)

22. Olfati-Saber, R. \& Murray, R. M. Consensus problems in networks of agents with switching topology and time-delays. IEEE Trans. Autom. Control 49, 1520-1533 (2004).

23. Lu, J. Q. \& Cao, J. D. Adaptive synchronization of uncertain dynamical networks with delayed coupling. Nonlinear Dynam. 53, 107-115 (2008).

24. Morelli, L. G. \& Zanette, D. H. Synchronization of Kauffman networks. Phys. Rev. E 63, 036204 (2001).

25. Jiang, L. L. \& Perc, M. Spreading of cooperative behaviour across interdependent groups. Sci. Rep. 3, 2483 (2013).

26. Molkenthin, N., Rehfeld, K., Marwan, N. \& Kurths, J. Networks from flows-from dynamics to topology. Sci. Rep. 4, 4119 (2014).

27. Wang, Z., Szolnoki, A. \& Perc, M. Optimal interdependence between networks for the evolution of cooperation. Sci. Rep. 3, 2470 (2014).

28. Ji, P., Peron, T. K., Menck, P. J., Rodrigues, F. A. \& Kurths, J. Cluster explosive synchronization in complex networks. Phys. Rev. Lett. 110, 218701 (2013).

29. Pérez, T., Garcia, G. C., Eguíluz, V. M., Vicente, R., Pipa, G. \& Mirasso, C. Effect of the topology and delayed interactions in neuronal networks synchronization. PloS ONE 6, e19900 (2011).

30. Lu, J. Q., Wang, Z. D., Cao, J. D., Ho, D. W. C. \& Kurths, J. Pinning impulsive stabilization of nonlinear dynamical networks with time-varying delay. Int. J. Bifurcat. Chaos 22, 1250176 (2012).

31. Lu, J. Q., Ho, D. W. C. \& Kurths, J. Consensus over directed static networks with arbitrary finite communication delays. Phys. Rev. E 80, 066121 (2009).

32. Zou, W., Senthilkumar, D. V., Zhan, M. \& Kurths, J. Reviving oscillations in coupled nonlinear oscillators. Phys. Rev. Lett. 111, 014101 (2013).

33. Li, R. \& Chu, T. G. Complete synchronization of Boolean networks. IEEE Trans. Neural Netw. Lear. Syst. 23, 840-846 (2012).

34. Li, R. \& Chu, T. G. Synchronization in an array of coupled Boolean networks. Phys. Lett. A 376, 3071-3075 (2012).

35. Yang, Z. D., Zhao, J. W., Li, R. \& Chu, T. G. General synchronization of multivalued logical networks. in Proc. 31st Chinese Control Conference, Hefei, Anhui, China pages 7717-7721 (2012).

36. Zhong, J., Lu, J. Q., Liu, Y. \& Cao, J. D. Synchronization in an array of outputcoupled Boolean networks with time delay. IEEE Trans. Neural Netw. Lear. Syst. (2014), doi: 10.1109/TNNLS.2014.2305722. 
37. Wu, Z. G., Shi, P., Su, H. Y. \& Chu, J. Exponential synchronization of neural networks with discrete and distributed delays under time-varying sampling. IEEE Trans. Neural Netw. Lear. Syst. 23, 1368-1376 (2012).

38. Hu, C., Yu, J., Jiang, H. J. \& Teng, Z. D. Exponential synchronization for reactiondiffusion networks with mixed delays in terms of $p$-norm via intermittent driving. Neural Networks 31, 1-11 (2012).

39. Silvescu, A. \& Honavar, V. Temporal Boolean network models of genetic networks and their inference from gene expression time series. Complex Syst. 13, 61-78 (2001).

40. Li, F. F. \& Lu, X. W. Complete synchronization of temporal Boolean networks. Neural Networks 44, 72-77 (2013).

41. Heidel, J., Maloney, J., Farrow, C. \& Rogers, J. A. Finding cycles in synchronous Boolean networks with applications to biochemical systems. Int. J. Bifurcat. Chaos 13, 535-552 (2003).

42. Goodwin, B. C. Temporal Organization in Cells. (Academic Press, London and New York, 1963).

\section{Acknowledgments}

The authors acknowledge the National Natural Science Foundation of China under Grant 61175119, Grant 61272530 and Grant 61203235, and IRTG 1740 (DFG and FAPESP). This publication was made possible by NPRP grant \#NPRP 4-1162-1-181 from the Qatar
National Research Fund (a member of Qatar Foundation). The statements made herein are solely the responsibility of the author[s].

\section{Author contributions}

J.L., J.Z., Y.T., T.H., J.C. and J.K. designed and performed the research, analyzed the results and wrote the paper.

\section{Additional information}

Supplementary information accompanies this paper at http://www.nature.com/ scientificreports

Competing financial interests: The authors declare no competing financial interests.

How to cite this article: Lu, J. et al. Synchronization in output-coupled temporal Boolean networks. Sci. Rep. 4, 6292; DOI:10.1038/srep06292 (2014).

This work is licensed under a Creative Commons Attribution-NonCommercialNoDerivs 4.0 International License. The images or other third party material in this article are included in the article's Creative Commons license, unless indicated otherwise in the credit line; if the material is not included under the Creative Commons license, users will need to obtain permission from the license holder in order to reproduce the material. To view a copy of this license, visit http:// creativecommons.org/licenses/by-nc-nd/4.0/ 